\title{
Lidil
}

Revue de linguistique et de didactique des langues

$41 \mid 2010$

Énonciation et rhétorique dans l'écrit scientifique

\section{Évolution des pratiques et des discours sur l'écrit à l'université : étude de cas}

\section{Christiane Donahue}

\section{(2) OpenEdition}

\section{Journals}

Édition électronique

URL : http://journals.openedition.org/lidil/3034

DOI : 10.4000/lidil.3034

ISSN : $1960-6052$

\section{Éditeur}

UGA Éditions/Université Grenoble Alpes

\section{Édition imprimée}

Date de publication : 30 mai 2010

Pagination : 137-160

ISBN : 978-2-84310-167-0

ISSN : 1146-6480

\section{Référence électronique}

Christiane Donahue, «Évolution des pratiques et des discours sur l'écrit à l'université : étude de cas », Lidil [En ligne], 41 | 2010, mis en ligne le 30 novembre 2011, consulté le 19 avril 2019. URL : http:// journals.openedition.org/lidil/3034 ; DOI : 10.4000/lidil.3034 


\title{
Évolution des pratiques et des discours sur l'écrit à l'université : étude de cas
}

\author{
Christiane Donahue*
}

\begin{abstract}
RÉSUMÉ
Dans le cadre états-unien des cours de «general education» (contenus généraux et transdisciplinaires) et des enseignements de «majeures » (contenus spécialisés dans la discipline), les étudiants travaillent entre le générique et le spécifique au niveau des savoirs, des discours, et des expertises. Les disciplines sont présentées comme des ensembles dynamiques de pratiques.

S'appuyant sur un double corpus de productions écrites et d'entretiens, l'étude établit un contraste entre l'évolution des pratiques d'écriture des étudiants, manifestant une forte implication dans les savoirs disciplinaires, et la faible «conscience discursive et disciplinaire» (Reuter et Lahanier-Reuter, 2008) que les étudiants manifestent lors des entretiens; ainsi, si l'on observe une lente évolution les amenant vers la pleine participation disciplinaire dans une «communauté de pratiques» (Wenger, 1999), on note également une difficulté à prendre conscience de cette évolution ou du moins à la relater verbalement.

L'étude nous interroge sur l'enseignement de l'écrit scientifique dans le cadre états-unien.
\end{abstract}

\section{ABSTRACT}

In the U.S. frame of "general education" and "majors", university students work between the generic and the specific at the level of knowledge, discourse, and expertise. Disciplines here are seen as dynamic sets of practices.

The article establishes a contrast between the evolution in students' writing practices, showing a strong sense of disciplinary embeddedness, and their less strong awareness and articulation of that same understanding of the relationship between writing and knowledge (Reuter and Lahanier-Reuter, 2008), that we find in their interviews. Thus, if we see a progression towards full participation in a "community of practice" (Wenger, 1999), we see as well a difficulty in becoming aware of

* Institute for Writing and Rhetoric, Dartmouth College, Hanover, États-Unis et THEODILE-CIREL. 
this progression, or at least difficulty articulating that awareness.

The study problematizes the teaching of university writing in the U.S.

L'écrit scientifique est perçu tant en France qu'aux États-Unis comme un mode puissant de penser et de produire les savoirs disciplinaires. Qu'il soit effectué par des chercheurs en sciences dures, humaines ou sociales, l'acte d'écrire n'est pas un acte d'écriture autour de ce qu'on a travaillé (Monroe, 2002), mais en fait un acte «d'écrire-construire le savoir», qu'il s'agisse de le communiquer aux autres ou de se l'approprier. Les types d'écrits que l'étudiant produit à l'université et les savoirs (disciplinaires et transversaux) qu'ils génèrent sont des éléments constitutifs du parcours de l'étudiant vers la maitrise de cet écrit scientifique, en ce qu'ils lui permettent de développer une expertise dans sa pratique des différents discours universitaires écrits (d'où le qualificatif d'expertise discursive que nous utiliserons dorénavant). Les établissements états-uniens qui se nomment «liberal arts $^{1} »$ constituent de ce point de vue un terrain d'étude privilégié pour étudier les relations entre savoirs transversaux et savoirs disciplinaires, expertise et types d'écrits produits : la structure du cursus comprend en effet des enseignements relevant d'une discipline (ou deux - les étudiants ayant le droit de suivre deux filières en même temps) et des enseignements transversaux relevant de la formation générale, non rattachés à une épistémologie disciplinaire. Cette dernière part du cursus est censée développer chez l'étudiant une diversité de perspectives et de savoirs sans spécialisation, et représente un tiers des enseignements dispensés.

Notre questionnement est axé sur l'évolution de l'écrit à l'université en lien avec l'évolution du savoir et comprend deux questions plus spécifiques : comment évolue l'expertise discursive dans sa relation aux savoirs transversaux et disciplinaires, et comment évolue la conscience qu'ont les étudiants de ces relations? Jusqu'à récemment aux États-Unis, rares ont été les études portant sur l'évolution de l'expertise discursive des étudiants au cours de leur cursus universitaire; la plupart des travaux se sont en effet axés sur les problématiques des étudiants entrant à l'université ou sur celles des étudiants en transition entre l'université et le monde professionnel. Certains travaux ont toute-

1. Il s'agit d'universités qui se focalisent sur un programme d'excellence pour les étudiants de premier cycle dans les matières traditionnelles des arts et des sciences. 
fois exploré l'écrit des étudiants en deuxième ou troisième cycle (voir par exemple Prior, 1998; Berkenkotter et Huckin, 1995). De mon point de vue, une des approches les plus productives pour cerner ces questions est celle des études longitudinales, de plus en plus effectuées aux États-Unis. C'est dans ce cadre que s'inscrit la présente étude.

Cet article s'appuie en effet sur une partie des résultats d'une recherche collective courant sur quatre années. Le groupe de chercheurs qui a mené cette étude a collecté et partiellement analysé la production écrite de 20 étudiants au cours de leur cursus de premier cycle dans une variété de disciplines, et a effectué des entretiens et recueilli des questionnaires en deuxième et quatrième année. Si la taille de l'échantillon n'autorise pas de généralisations, elle permet en revanche de dégager des observations indiquant des directions de recherche futures. Ici, la confrontation entre l'analyse textuelle des écrits (axée plus spécifiquement sur l'intertexualité et sur le positionnement de l'étudiant-auteur) et l'analyse des entretiens nous informe sur les points de convergence et de divergence entre les pratiques d'écriture universitaire et les discours que les étudiants tiennent sur ces pratiques; ces observations nous permettront ainsi d'identifier certaines caractéristiques de l'évolution de l'expertise discursive de ces étudiants au cours de leur cursus. Afin d'illustrer mon propos, trois cas particuliers seront exposés, celui d'un étudiant en philosophie, un en biologie/sciences politiques, et un en psychologie.

\section{L'expertise discursive et les disciplines : quel cadre théorique?}

Les réflexions autour de l'écrit universitaire et de la disciplinarité entrainent une réflexion sur les représentations générales des disciplines et des genres, et sur l'identification de la façon dont les étudiants gèrent le savoir disciplinaire et les discours d'une discipline. Dès les années 1970, le champ de «composition studies» aux États-Unis, a eu tendance à considérer «l'expertise» comme étalon-or auquel le novice aspire. Comment théoriser cette expertise ?

Les recherches en linguistique et en composition analysent l'écrit expert de différents domaines scientifiques afin d'en identifier les caractéristiques (voir par exemple Swales, 1990; Hyland, 1996; Fløttum, 2008; Rinck, 2006; Bazerman, 1988; Geisler, 1994). Ces caractéristiques ne sont pas statiques; elles évoluent avec les travaux menés dans le champ disciplinaire concerné, ainsi qu'avec l'évolution des médias et 
des outils. Elles ne définissent pas le champ, bien qu'elles servent souvent à aider les usagers à reconnaitre un genre donné (tel que l'article par exemple), c'est-à-dire une activité discursive récurrente, caractérisée, et qui invite à un type de lecture spécifique. Ces caractéristiques sont également polyvalentes, fluctuantes, reconfigurées pour et par chaque genre.

Comme le genre, la discipline n'est pas un objet fixe avec des caractéristiques stables, mais elle comprend au contraire un ensemble dynamique et fluctuant de pratiques relevant de la littéracie, pratiques qui construisent la discipline et se trouvent construites par elle (voir Anson, 1988 ; Langer, 1992). Le cheminement des étudiants au sein d'épistémologies bien spécifiées à travers les enseignements disciplinaires mais aussi dans le cadre de leur formation générale est ainsi au cœur de la question de l'expertise discursive. Les études longitudinales comme genre d'étude montrent en général que le modèle de développement de cette expertise est complexe. En particulier, on voit que les modèles linéaires d'expertise ne rendent compte que d'une partie de l'histoire de la maitrise progressive et complexe de l'écrit. Sommers et Saltz (2004) montrent, par exemple, comment les étudiants ayant quitté leurs études secondaires en position d'«expert» doivent redevenir novices à l'université, une perspective également soutenue par Hjortshoj dans ses travaux portant sur la transition entre le secondaire et l'université (2001). Plus récemment, Gannett (2008) fait l'hypothèse que ce statut est en fait partagé par tout scripteur entrant dans un nouveau contexte. Nous ne pouvons ainsi étudier les écrits universitaires sans étudier les activités et les contextes dans lesquels ils sont enracinés. De plus, certaines études longitudinales suggèrent que les genres produits à l'université ne sont pas toujours liés aux genres experts. L'activité n'est pas équivalente; la réception est également différente.

\section{L'évolution des cadres théoriques états-uniens concernant l'expertise discursive}

Il existe des cadres théoriques nous permettant de prendre en compte le statut de l'étudiant et de son expertise discursive en évolution : on leur empruntera en particulier les termes d' «orientation» (François, 2009) et de «mature practices» (Lave et Wenger, 1991), terme qui renvoie à des pratiques illustrant une certaine maturité de l'étudiant vis-à-vis des caractéristiques de l'écrit exigées dans sa discipline, que l'on traduira dorénavant, faute de mieux, par «pratiques maitrisées». Plutôt qu'une entité stable aux savoirs et conventions bien établis, une communauté 
de pratiques est «un ensemble de relations entre personnes, activités, et mondes, dans le temps, en relation parfois tangentielle, parfois superposée avec d'autres communautés» (Lave et Wenger, 1991, p. 135). Dans ce cadre, les disciplines elles-mêmes sont envisagées comme conflictuelles, glissantes, fluctuantes, des «réseaux ouverts, forgés dans l'activité relationnelle» des participants (Prior, 1998, p. 25). Les disciplines sont humaines, et non des «structures homogènes anonymes de conventions linguistiques, rhétoriques, et épistémiques » (Prior, 1998, p. 22). Pour Lave et Wenger (1991), l'apprentissage est toujours une activité située, caractérisée par la possibilité d'une pleine participation dans la communauté de pratiques en question, mais également d'une «participation périphérique légitime» qui définit le statut des participants qui cheminent vers les pratiques maitrisées. Chaque pleine participation entrainera des capacités tout à fait particulières aux savoirs en question, à la nature de l'expertise, et à la possibilité de s'adapter aux contextes toujours changeants.

À travers la notion d'orientation, développée par François (2009), on envisage les activités discursives comme activités de navigation entre le commun et l'individu, le social et l'individuel. Pour François, le terme d'orientation offre une façon de comprendre les activités discursives comme activités d'interaction entre l'individu et l'autre, donnée indirectement par divers modes de représentation (2009, p. 11). L'étudiant se situe, s'oriente, en l'occurrence vis-à-vis des autres textes, des idées et des voix d'une discipline, et cette plongée dans la culture disciplinaire est directement corrélée à ses performances à l'écrit, ainsi que l'a montré l'étude longitudinale de Sommers et Saltz (2004). Ces mouvements sont, selon Carter (1990), le tissu d'une théorie pluraliste de l'expertise comme, simultanément, une application de stratégies globales comme point de départ, en particulier dans des contextes qu'on aborde pour la première fois, et un développement de stratégies locales qui deviennent naturalisées, «des performances fluides qui ne sont que rarement analytiques, délibératives, conscientes »(Carter, 1990, p. 272). Les stratégies globales servent à s'orienter une première fois, mais les stratégies locales, contextuelles, permettent d'autres orientations.

L'évolution de l'expertise discursive a également été liée au rôle d'un travail de réflexion «méta», que ce soit à l'écrit ou à l'oral (protocoles, consignes de réflexion, questionnaires...), pour les étudiants. Ce travail est reconnu dans beaucoup de recherches : selon les recherches en «transfert des savoirs» (voir par exemple Salomon et Perkins, 1989; Alexander et Murphy, 1999; Tuomi-Grohn et Engestrom, 2003), l'apprentissage qui fonctionne est un apprentissage que l'étudiant peut 
verbaliser, et selon les recherches en acquisition des savoirs (Boyer et Delbrayelle, 2009; Bangert, Hurley et Wilkinson, 2004), on ne «sait» pas ce qu'on ne peut pas «faire» ou dire. L'aspect de la verbalisation, une activité associée à un travail «méta», nous interroge sur le développement de pratiques maitrisées citées ci-dessus : faut-il en être conscient?

L'exploration de la relation entre disciplinarité, savoirs et écrit chez les étudiants est complexe. Les questions principales sont les suivantes : comment avoir accès à l'évolution des étudiants, leurs manières de participer, leurs prises de consciences, leurs prises de position, leurs productions? Comment se servir d'outils d'analyse nécessairement statiques pour explorer des pratiques d'écriture qui, selon le cadre adopté, sont fluctuantes? Autrement dit, comment l'expertise en devenir peutelle être décrite?

\section{Méthodologie}

Notre analyse compare des observations tirées d'un corpus de textes et d'entretiens menés avec leurs auteurs.

Le corpus est ainsi constitué d'un groupe de textes et d'entretiens tirés d'un plus grand corpus. Notre étude longitudinale s'appuie sur des écrits très divers réalisés par vingt étudiants en dix disciplines différentes durant les quatre années de leur cursus «undergraduate» : écrits formels, informels, brouillons, examens, écriture créative, rapports de stages, écrits scientifiques, posters, projets de recherches, etc. L'analyse de cas présentée ici prend appui sur deux séries de textes nommées respectivement «analyse» et «recherches» de trois étudiants qui nous ont semblé être des cas intéressants et représentatifs de l'ensemble, en ce qui concerne leur façon d'évoluer par rapport à l'écrit et aux savoirs disciplinaires. L'écrit d'«analyse» est ici défini comme un écrit qui doit présenter une perspective argumentée autour de lectures ou contenus de cours travaillés en classe; l'écrit de «recherches» est un écrit appuyé en majeure partie sur des lectures ou des expériences en dehors de la classe, indépendantes, et exigeant une synthèse. À noter que pour la taxinomie de Bloom (1956), reprise ici, le travail de synthèse, qui incorpore un travail d'analyse, est plus exigeant que le travail d'analyse. En outre, l'écrit de recherches s'inscrit davantage dans une discipline donnée en ce qu'il doit respecter les spécificités disciplinaires.

Le corpus dans son ensemble a été recueilli de 2004 à 2008, et comprend textes écrits, entretiens, et questionnaires. Les étudiants impli- 
qués ont choisi de participer ${ }^{2}$; sur les 38 étudiants qui se sont portés volontaires, 20 ont accepté au final de jouer le jeu, dont 16 ont fourni tous les types de textes requis sans sauter de semestre. L'établissement est une université de petite taille (2000 étudiants) avec une très bonne réputation nationale (l’Université de Maine-Farmington) concernant la qualité de son premier cycle. À la fin de chaque année, les étudiants ont répondu à un questionnaire. À la fin de la deuxième et de la quatrième année, ils ont participé à des entretiens d'une demi-heure, avec les mêmes questions chaque année; ces entretiens ont été transcrits. Pour cet article, je m'appuie sur trois cas représentatifs, inscrits respectivement en philosophie (Allen), biologie (Joëlle), et psychologie (Robert) (appartenant ainsi aux trois grands domaines, «humanités», «sciences dures», «sciences sociales»), en notant que les étudiants en biologie et en psychologie sont soit en «double majeure» soit en «majeure/mineure», suivant en même temps la filière de sciences politiques.

\section{Analyse des textes}

Les textes écrits font l'objet d'une analyse sous les angles suivants : intertextualité, argumentation, organisation, positionnement du sujet, orthographe/syntaxe. Un groupe d'enseignants-chercheurs a été formé pour l'analyse ${ }^{3}$; leurs lectures ont été vérifiées par une relecture d'un échantillon effectuée par l'auteur. Ces analyses textuelles utilisent des indices établis par Donahue (2002, 2005, 2008), focalisés sur l'énoncé (Bakhtine, François) et sur les perspectives de la théorie de l'activité (Prior, 1997; Bazerman, 1988; Russell, 1996; Tuomi-Grohn et Engestrom, 2003). Ces indices cherchent à mettre en évidence des éléments indicatifs des pratiques maitrisées et des façons de s'orienter des étudiants. Notamment, la gestion de l'intertextualité est généralement acceptée comme indice de succès dans la navigation de la polyphonie discursive, ainsi que d'un engagement de la part de l'étudiant

2. Ils ont été pour cela rémunérés : de petits cadeaux de temps en temps, des prix, et de l'argent pour chaque document rendu ( 25 centimes pour chaque texte informel, 50 centimes pour chaque brouillon, un dollar pour chaque texte formel - essai, examen, dissertation, etc.).

3. Rebecca Berger, Stephan Desrochers, Marcia Nash, Steven Pane et Kristina Wolff, formés par une équipe d'enseignants-chercheurs de cinq disciplines (sociologie, sciences de l'éducation, linguistique, psychologie et musique). 
dans les discussions, les débats, les «voix» de la discipline en question (Boch, 1999; Boch et Grossman, 2001; Daunay, 2002; Reuter, 2001; Delcambre, 2001 ; Fabre-Cols, 2001; Guibert, 2001; Rinck, 2006; Howard, 1999; Donahue, 2005; Donahue, 2007); le positionnement du sujet est un indice d'orientation.

Les entretiens ont fait l'objet d'une analyse de contenu («content analysis») et d'une analyse des isotopes de sens, qui seront mises en parallèle avec l'analyse de l'intertextualité et du positionnement de l'auteur-étudiant dans les deux types de textes («analyses» et «recherches»).

\section{Résultats}

On présentera d'abord les caractéristiques communes aux trois sujets pour dégager ensuite ce qui fait leurs spécificités; nous rappelons que les questions posées par l'analyse de ces trois cas se retrouvent pour une large part dans l'intégralité de notre corpus, composé de 20 sujets.

\section{Caractéristiques textuelles communes}

Les caractéristiques communes textuelles peuvent être réparties en quatre grands domaines : quantité, rythme, et types d'écrits; façons d'interagir avec les sources; façon de s'introduire et de se représenter dans l'écrit; facilité avec la terminologie disciplinaire.

\section{Quantité, rythme, type}

Malgré la nature de l'institution en question, dans une université «liberal arts» (focalisée sur les arts et les sciences et non la professionnalisation), les étudiants n'ont pas produit beaucoup d'écrits dans l'ensemble, entre 100 et 170 textes tout compris par étudiant sur quatre années. Parmi ces écrits, informels et formels, $19 \%$ correspondent aux types «analyse» et $35 \%$ sont des écrits dits de «recherches». Au fil de leur cursus, les étudiants ont produit de plus en plus de textes écrits jusqu'à l'avant-dernier semestre, avec une réduction pendant le dernier semestre. La longueur des textes a évolué à travers les quatre années, de 2-3 pages en première année vers une vingtaine de pages en quatrième année. Cela dit, dans les trois cas présentés ici, on trouve davantage de productions de textes analytiques en philosophie, davantage d'écrits de recherches en biologie, psychologie, et sciences politiques, et plus généralement un plus grand nombre de textes produits en philosophie et en psychologie/sciences politiques. 


\section{Interactions textuelles}

L'évolution dans ces interactions se voit davantage dans les écrits de recherches que les écrits analytiques. La façon d'introduire les sources évolue, les étudiants adoptant dans les premières années des verbes introducteurs de discours rapporté véhiculant une information minimale («says», «states», «writes») pour utiliser par la suite des verbes orientant davantage le point de vue argumentatif de l'auteur cité («argues», «supports», «critiques»). Parallèlement, on observe un nombre croissant de sources référencées au fil du cursus. Enfin, de la première à la dernière année, les étudiants passent d'un mode de référencement essentiellement axé sur la citation directe à la reformulation phrastique ou synthétique du discours d'autrui, ce dernier mode correspondant davantage aux pratiques expertes (Rinck et al., 2007).

Une des évolutions les plus nettes dans l'écrit des étudiants est celle de leur maitrise dans l'interaction avec d'autres textes dans leurs écrits de recherches. La gestion de leur pensée par rapport à la pensée des autres est perçue dans les taux de citations et de paraphrase, et dans les taux de marqueurs de cohérence permettant d'intégrer ce discours d'autrui. En première année, l'usage de citations est élevé, de 16 à 29 pour mille mots, alors qu'en quatrième année ce taux diminue, de 6 à 4,7; de même pour les paraphrases, dont le nombre s'élève en première année de 7 à 31 pour mille mots, et en quatrième année, de 2,8 à 8 pour mille mots, à l'exception de l'écrit de recherches d'un étudiant qui fait l'inverse (passant de 0 à 15 paraphrases). Le taux de marqueurs explicites de cohérence s'accroit de façon considérable, d'une moyenne de 10 pour mille mots en première année à une moyenne de 18 en quatrième année. Pour les écrits d'analyse, ces éléments restent plus stables. En première année, ce taux était de 4 à 14 citations pour mille mots de texte, et en quatrième année, de 1,2 à 6,5 citations pour mille mots; pour les paraphrases, en première année entre 7 et 10,5 paraphrases pour mille mots, et en quatrième année, de 4 à 8 paraphrases pour mille mots. Quant aux connecteurs explicites, les écrits d'analyses sont restés stables avec environ 17 marqueurs pour mille mots.

\section{Introduction/représentation du sujet}

Les interactions textuelles sont en corrélation avec une autre évolution de la première à la dernière année : en première année, les étudiants semblent être mal à l'aise avec une représentation/intégration textuelle de soi, de la voix du soi, qui est la plupart du temps évacuée du texte au moyen de stratégies linguistiques telle que la voix passive. En quatrième 
année, ils semblent à l'aise avec diverses formes de mouvement entre soi et les voix des textes (je, nous, les noms d'auteurs, etc.).

\section{Terminologie disciplinaire}

Les écrits offrent de multiples exemples en quatrième année d'emplois d'une terminologie liée à la discipline en question («bureaucratic tribalism», «particular primate taxa», etc.) qu'on ne trouve pas en première année et qui sont employés de façon appropriée.

\section{Analyse des entretiens : caractéristiques communes aux trois cas étudiés}

Les caractéristiques communes des discours sur l'écrit et le savoir, détaillées ci-dessous, relèvent de quatre domaines : les représentations du savoir; les représentations de l'écrit disciplinaire par rapport à l'écrit produit dans les cours d'éducation générale; la facilité d'expression concernant l'écrit; la reconnaissance du rôle de la nouveauté et de l'importance d'une souplesse rhétorique. Ces caractéristiques ne semblent pas toujours refléter ce qu'on trouve dans les écrits.

\section{Représentations du savoir}

À l'issue de leur cursus, les étudiants se représentent très classiquement le savoir comme un objet que l'écrit permet avant tout de conserver. Pour Joëlle, par exemple, en biologie, «Writing things down and really thinking about your writing really makes you remember stuff better, so I know I won't forget certain elements of the classes that I'm taking... ${ }^{4} »$; pour Robert, en psychologie, «it kind of imprints it [the knowledge] and makes you know it even more» (l'écrit capte le savoir). Écrire les contenus est un acte permettant de créer un dépositaire du savoir permanent auquel il peut toujours avoir recours : «as a learner, you sort of have the brain connections that get disconnected once you're done taking the class... if I had that paper I could read it over and really remember what I did... instead of having them [the ideas] lost forever ». Dans le même esprit, Allen, en philosophie, souligne que le savoir est transcrit et sauvegardé par le fait de l'écrire; on peut ainsi le retrouver plus facilement : "If I tried to figure out, what was that reading, I can look at the paper I wrote about it.»

4. Les extraits de textes et d'entretiens des étudiants ont été laissés dans la langue d'origine afin de préserver les nuances des formulations. 
Pour les étudiants, l'écrit constitue également une forme de preuve que les apprentissages ont bien eu lieu et que les contenus sont maitrisés; l'écrit est assimilé à un outil de supervision : «writing serves to make sure you've done the necessary work; to make sure you did the reading; to make sure the professor knows you understand the material» (Allen); «it's more of a test to see that you've, you know the, you know what you have to know, you've done the work, you've done the reading» (Robert). Robert lie l'écrit aux savoirs ${ }^{5}$ alors qu'Allen ne souligne que le besoin de montrer qu'on a fait le travail demandé.

\section{Représentations de l'écrit}

Les étudiants se représentent les attentes autour de l'écrit différemment dans les cours en éducation générale et dans ceux de leurs disciplines. En première et deuxième année, ces différences ne sont que rarement évoquées au plan des conventions (excepté la question des normes autour de l'usage du «je» et des citations), mais plutôt au plan de la construction des savoirs : quels sont les faits jugés acceptables pour soutenir une assertion, quels types de présupposés je peux envisager, quels types de jugements je peux émettre par rapport à une ligne théorique donnée, etc.

Pour Allen,

I thought I was expected [in a freshman history course] to agree with the material that was given, we had to read a book and then write a paper, and I sort of expected to have to agree. [...] you had to disagree with what was given and you had to use educational, obviously well written responses and arguments against it.

Au départ, Allen s'est mis en position de devoir s'accorder aux voix de la discipline, mais au fur et à mesure il cherche à s'engager avec, voire s'opposer à, ces voix. Joëlle spécifie que «scientific writing is, to do it well is really difficult and it takes lots of practice [...] conducting our own research but then having to put findings, and writing statistical analyses, being able to interpret it concisely and effectively is hard», et que les normes et les attentes dans les cours d'éducation générale sont nettement moins exigeantes : «I can talk much more in the first person in different types of writing [...]; personal reflection and things like that.» Les étudiants identifient les disciplines en partie par les pratiques

5. Lors de l'entretien, Robert utilise le mot «preuve» 12 fois dans sa réponse à la question suivante : «Quel rôle l'écrit joue-t-il dans vos études?» 
d'écriture qui y sont convoquées. Ainsi, Allen indique qu'il a choisi la filière de philosophie parce que cela ressemble aux études de Lettres, «it [my interest in English] kind of morphed into philosophy for me... which is just a lot of reading and writing anyway». Il semble définir la discipline par ses activités de lecture/écriture plus que par ses particularités épistémologiques.

En quatrième année, les perspectives des étudiants sont mieux définies et avec plus de maturité; ce constat est à mettre en parallèle avec les progrès perceptibles dans leurs écrits, mais - et c'est essentiel - les représentations des étudiants concernant l'écrit et le savoir ne se modifient pas fondamentalement.

Les thèmes avancés, disciplinaires, sont évoqués avec plus de facilité, et on observe une tendance à considérer le savoir comme un puits sans fond - trop à dire, trop d'idées à gérer - (Allen par exemple dit «It was hard not writing a book on it...»); parallèlement émerge la prise de conscience d'une difficulté à travailler l'écrit de façon synthétique et à faire le deuil de l'analyse de détails (pour Robert, «how to fit together all those pieces...» et pour Joëlle, «the level of analysis I'm expected to do is much more based on numbers and proof and not being allowed to say certain things if there's not at least one little detail to back it up»).

Les deux «scientifiques» (Joëlle, biologie, et Robert, psychologie) rendent compte dans leur entretien d'une distinction entre deux genres scripturaux relevant de l'écrit de recherche - revue de la littérature vs recherches expérimentales. Joëlle est particulièrement claire à ce sujet : «A good chunk of what I have to write are research papers for any of my classes, my own research or just looking at other people's studies.»

\section{Facilité d'expression concernant l'écrit}

Pour les étudiants, les difficultés rencontrées à l'écrit sont toujours liées aux questions de nouveauté et de non-familiarité : "It was the first time I'd ever encountered a lot of material; it was something I'd never done before; I wasn't expecting it.» En particulier, Joëlle raconte une expérience lors d'un semestre dans une autre université concernant la construction des savoirs et l'impression d'appartenir ou non à une communauté. Elle décrit la difficulté ressentie avec certains genres écrits lors de son cours de théorie politique, en termes de nonfamiliarité, de manque de préparation, et d'externalité aux normes et attentes d'une communauté établie : "I didn't have my community base», évoque-t-elle. 


\section{Reconnaissance du rôle de l'écrit}

Les étudiants soulignent, en quatrième année, qu'il faut avant tout une souplesse rhétorique, le besoin selon Robert d'être «loosened up», de reconnaitre que chaque contexte fait appel à ses propres styles, le besoin d'être «malleable» (Joëlle), et que chaque cours, thème, matière, niveau fait appel à différentes normes et attentes.

\section{Caractéristiques spécifiques aux trois sujets étudiés}

L'expérience individuelle des étudiants (dont on trouvera les moments clés synthétisés dans un tableau en annexe) nous offre une autre façon de comprendre l'interaction entre l'écrit et les perceptions rapportées dans les entretiens.

Dans l'ensemble, les trois cas, par leurs aspects communs et leurs particularités, nous offrent des façons de comprendre les évolutions productives et les écarts entre compétence scripturale et conscience méta-scripturale. Cette conscience méta-scripturale est constituée d'une conscience disciplinaire, telle que décrite par Reuter et Lahanier-Reuter (2008) et d'une conscience discursive. À travers leurs productions écrites réalisées tout au long de leur cursus (et en particulier dans les écrits de recherche), les étudiants montrent en effet une connaissance progressive et de plus en plus solide des habitus de la discipline en matière de littéracie, qui concernent non seulement la façon dont un auteur interagit à l'écrit avec d'autres textes ou d'autres idées, avec les savoirs disciplinaires, mais aussi le positionnement du sujet par rapport à autrui. En revanche, dans les entretiens, on note une difficulté persistante à verbaliser ces progrès, comme si l'acculturation à la discipline se faisait sans qu'ils en aient conscience. En ce qui concerne les écrits de recherche, l'évolution des pratiques vers l'expertise reflète l'appropriation des habitus de la discipline de référence et une bonne compréhension de ce qui fait son autorité. En revanche, pour les écrits d'analyse, l'évolution est moins nette, soumise à des régressions ponctuelles et des formules reproduites.

\section{Pratiques expertes discursives et relations aux savoirs}

La relation des étudiants au savoir se présente différemment dans les différentes disciplines. Pour Allen, en philosophie, la lecture et l'écriture deviennent indissociables. En deuxième année, il les perçoit comme activités linéaires : on lit et puis on écrit («understand[ing] the reading and show [ing] that you demonstrate that and then from there just 
put[ting] your own stuff into it»). En quatrième année, la lecture comprend les actes de reformulation, d'appropriation et d'intégration, en fonction des lectures en cours mais également en dehors des cours : " "earlier [in college] it was more just synthesizing everything you had done in the semester for the papers" while "later on in college I've had to do more independent research".» Par rapport aux autres disciplines expérimentées dans des cours d'éducation générale, la philosophie exige qu'il «aille plus loin»; les autres cours demandent de produire des réponses plus directes, des résumés ou des écrits n'exigeant pas de fournir une réflexion approfondie.

De même, la relation au savoir évolue, quand on l'analyse à travers l'usage de citations. Ce qui en première année est utilisé comme outil global et conventionnel («il faut citer...») est devenu en quatrième année une façon de se positionner et de s'orienter par rapport aux voix et aux idées des auteurs disciplinaires, mais cette évolution apparait davantage dans les écrits de recherches que dans les écrits analytiques. Pour Allen, par exemple, la valeur «universelle» des citations n'existe plus : "I use a lot of quotations when I write, which can either be a strength or really not depending on what type of writing it is and how I'm doing it.» Dans ses écrits de recherche, les pratiques et les positionnements semblent bien différents : l'idée force est complexe, les sources sont intégrées et servent à soutenir cette idée, les transitions tissent la cohérence entre les sources et la voix de l'auteur, et les notes de bas de page signalent aussi un positionnement dans le champ.

Pour Robert, cette modification se trouve dans l'évolution, dans ses écrits de recherches, de son positionnement et de l'interaction avec les sources. En première année, le report linéaire des sources, chacune introduite par des verbes «être» modalisés («semblent être»), est frappant, en parallèle avec un usage massif de citations (jusqu'à 55\% du texte). À la fin de ses études, son projet final est interdisciplinaire (en linguistique et en psychologie). Il produit une analyse du style de ses propres écrits en fonction des textes qu'il a lus : «My honors thesis is an attempt to demonstrate the structural and emotional changes that can occur in one's writing after reading particular authors.» Dans son texte, il met en œuvre des stratégies scientifiques, telles fournir des définitions, offrir une discussion de la validité scientifique des outils mis en place, intégrer des tableaux, ou utiliser un vocabulaire d'analyse (le fait de parler par exemple d'une «congruence particulièrement robuste»). L'interprétation et l'analyse «méta» sont davantage du côté des disciplines humanistes; considérons par exemple la phrase d'introduction 
de son projet: «In order to begin writing this report, I am obliged to consider the problem of 'appropriate ways to begin a report'.»

On voit dans les écrits de recherches une évolution frappante par rapport à la forme et aux savoirs engagés. $\mathrm{Si}$, en première année, pour Joëlle, le genre du «lab report», très normé, contraint le produit et ne permet l'appui que sur une seule source, en quatrième année, le rapport scientifique final comprend 45 références, bien tissées dans le texte. Les connecteurs sont plus fréquents et plus appropriés; l'encadrement rhétorique est également plus fréquent : "While the natural world we observe is a complex system, some aspects of it are easy to quantify regardless of variation.»

Les textes d'analyse ne suivent pas le même chemin. En première année, les textes analytiques de Joëlle se focalisent sur les savoirs et l'autorité des autres («I found that Reiman did a good job of summing up some of my feelings about this statement when he wrote...»); en quatrième année, malgré des modifications en syntaxe, on observe une évolution dans la diversité des sources et dans sa relation au savoir, perceptible dans le fait qu'elle introduit des notes de bas de page (outil assez sophistiqué); les analyses produites restent en déférence aux auteurs lus et aux savoirs qu'ils proposent. Le travail textuel demandé aux étudiants dans les analyses semble ne pas beaucoup évoluer au cours des quatre années : il s'agit principalement de rendre compte des savoirs exposés dans leurs lectures et du point de vue de leurs auteurs. Joëlle, par exemple, soutient et complète les perspectives des auteurs : "I found this article to be very well thought out», modalisé comme «undoubtedly valid...». Ces mêmes motifs se retrouvent en quatrième année, mais dans un registre davantage académique («well thought out» devient «is a noteworthy voice of reason», par exemple). Les verbes qui introduisent l'auteur, en quatrième année, ne sont plus de l'ordre de «il croit» ou «il dit» mais «il crée», «il maintient», «il reconnait», ou «il fait croitre sa crédibilité». L'ensemble de ses analyses s'articule avec des connaissances plus étendues sur des thématiques et des auteurs représentés dans sa discipline, et on note un équilibre entre reprise et extension des idées des autres.

\section{Le dit vs l'écrit}

Les écarts entre représentations et pratiques scripturales sont intéressants, notamment en ce qu'ils permettent de questionner le rôle de la métacognition. Robert ne change pas, à l'oral, son discours sur les fonctions de l'écrit au fil de son cursus dans la discipline; de la première à 
la quatrième année, sa perception de l'écrit reste techniciste : «in high school, you can make things up pretty easily, and you can't do that in college, [you] have to read in order to know what [you are] talking about.» Les écrits courts sont «just written» sans planification ou révision, parce que le savoir peut être tenu en mémoire; les écrits longs construisent des savoirs complexes et exigent des étapes d'écriture et de réflexion. Cette représentation de l'écrit comme moyen de disposer d'un «savoir sur papier» se retrouve dans la méthode décrite par Robert de transcription des idées sur des petites fiches: «I don't have to scroll [down the computer screen] or anything, it's [the knowledge] just right there.» Or, dans ses pratiques, on l'a vu, l'écrit connait au cours des quatre années du cursus des évolutions considérables, qui témoignent d'une représentation de l'écrit qui va bien au-delà du simple rôle d'aide-mémoire.

Une autre relation aux savoirs s'exprime chez Joëlle, qui expose les avantages que revêt l'écrit pour ceux qui ont du mal à s'exprimer en cours et qui peuvent se réfugier dans l'écriture, notamment dans la perspective de se préparer à l'oral : «I think it's meant to prepare you to be able to get your thoughts down on paper so you can be more articulate when you speak, [...] to externalize thinking, to get ideas down and feel comfortable to bring it out and discuss it.» Plus globalement, la fonction prioritaire de l'écriture réside dans la possibilité de «dire» le savoir, que ce soit à l'écrit ou à l'oral.

\section{Le cas de l'éducation générale et de multiples «majeures»}

Ces différentes observations interrogent sur le cursus universitaire états-unien et sa façon de complexifier le développement de l'expertise discursive. Robert indique dès sa première année que différents domaines disciplinaires appellent différentes approches de l'écrit : l'écrit en «Political Science» est «very fact-based writing which is why you have to do all the reading and you even have to do extra stuff, like reading the newspaper [...], to do extra to make it part of your real life and not just study». En quatrième année, Robert exprime le besoin de connaitre les conventions liées aux savoirs : «I need to work harder when it's not something I'm familiar with [...]; you need to be more cognizant of the information.» À cela s'ajoute une conscience des attentes et des activités, perçues comme variables en fonction des professeurs (attitude très liée aux premières années d'études), mais aussi en fonction des contenus disciplinaires : «I'm sure [Professor A] would 
expect different things in her Research Methods course and her Counseling class.»

Ces relations se présentent chez Joëlle d'une autre façon. À la fin de ses études, elle affirme : «I just find that my writing in my science classes is a lot more restricted than... I just can't make assumptions at all in my papers, at all. In that sense they are a lot different from my political science classes where I can generalize a lot more.» Les cours d'éducation générale lui auraient permis en particulier de prendre conscience des différences au plan des conventions de l'écrit selon les disciplines :

writing is just different, I've had to kind of, ah, cite citations and stuff in scientific writing is different than my political science writing. Just kind of like getting used to those two formats. I actually messed up on one of my science papers. In a couple of my citations I put it in political science format because I was writing, working on two papers at the same time that day, so I messed up a little bit that way, but yeah, that's one of the things, just the, um, how they expect you to prove your research and things like that, it's different.

On voit bien dans ce commentaire le décalage entre les représentations et les pratiques de Joëlle.

\section{Conclusions}

Les questions posées par ces observations autour des différences et des aspects partagés, bien que préliminaires et limitées, offrent certaines pistes interprétatives :

- dans les écrits «de recherche», la relation entre évolution des savoirs disciplinaires et expertise discursive est étroite (et permet ainsi le rejet d'une version «autonome» d'expertise à l'écrit). Mais cette expertise semble passer par des stratégies globales pour aboutir à des pratiques maitrisées locales, ainsi qu'à une souplesse rhétorique, telle que proposée par Carter (1990);

- le système d'éducation générale aux États-Unis peut rendre plus compliqué le processus de développement discursif pour les étudiants, bien qu'il rende possible davantage d'adaptabilité au niveau des conventions ;

- les étudiants s'orientent vis-à-vis du savoir dans leurs disciplines différemment dans leurs verbalisations en entretien par rapport 
à leurs productions écrites, ce qui soulève des questions importantes, notamment en ce qui concerne le va et vient entre éducation générale et disciplines ou entre disciplines.

Cet ensemble de constats nous interroge sur le décalage potentiel entre l'évolution des compétences scripturales dont ont fait preuve nos trois sujets au cours de leur cursus et la relative pauvreté de leur conscience méta-scripturale, ou du moins leur capacité réduite à verbaliser leurs représentations de l'écrit et de ses fonctions dans la discipline. Dans ses analyses des étudiants de deuxième cycle, Prior (1998) suggère que les étudiants négocient en permanence entre différents réseaux disciplinaires. Il souligne que l'hétérogénéité des types d'écrits demandés aux étudiants peut nuire à leur développement de pratiques maitrisées d'expertise discursive. Cette question est d'autant plus pertinente dans le cas d'un modèle d'éducation supérieure tel que celui en vigueur aux États-Unis, qui mélange spécialisation et généralisation, accentuant ainsi cette hétérogénéité en premier cycle.

Le contexte étudié ici nous permet de penser également que certains types d'écrits sont plus formateurs que d'autres pour conduire à des pratiques maitrisées. En effet, les caractéristiques des pratiques scripturales expertes et constitutives de la discipline semblent progressivement perçues et maitrisées dans les textes d'étudiants, mais ne s'accompagnent pas nécessairement d'une verbalisation raisonnée de ces pratiques ${ }^{6}$. Les textes manifestent ainsi une évolution qui semble peu conscientisée dans les entretiens; comme on l'a vu, on trouve dans les textes une aisance croissante dans le positionnement de soi et dans la relation avec le discours d'autrui, ainsi qu'une facilité dans l'usage de la terminologie en vigueur dans la discipline. Toutefois, on a observé dans les entretiens une évolution dans les représentations de la complexité de l'écrit universitaire et des attentes relatives à la discipline et aux savoirs qui la constituent, qui dépassent la simple réponse aux exigences (voire aux caprices) d'un professeur individuel. Le rôle que peut jouer l'alternance des cours d'éducation générale et des cours disciplinaires a été simplement évoqué ici. Une des questions qui restent à explorer est ainsi celle du rôle du double cursus états-unien, et de son

6. Cette question des normes et des exigences de l'éducation générale par rapport aux disciplines pourrait bien sûr permettre de souligner des différences dans les cours ou chez les étudiants qui expérimentent différemment en fonction de leurs investissements. 
influence dans l'évolution des pratiques scripturales et de la conscience méta-scripturale des étudiants. Les résultats de l'étude plus large apporteront, nous l'espérons, des compléments d'information en nous montrant d'autres caractéristiques spécifiques des textes non-disciplinaires et disciplinaires, que nous mettrons en perspective avec les discours des étudiants sur ces pratiques.

\section{RÉFÉRENCES BIBLIOGRAPHIQUES}

Alexander P. A. et Murphy P. K. (1999) : «Nurturing the seeds of transfer: a domain-specific perspective», International Journal of Educational Research, vol. 31, p. 561-576.

Anson C. (1988) : «Toward a multidimensional model of writing in the academic disciplines», dans D. Jolliffe (éd.), Writing in academic disciplines, Norwood, NJ, Ablex, p. 1-36.

Bangert R., Hurley M. et Wilkinson B. (2004) : «The effects of schoolbased writing-to-learn interventions on academic achievement: a metaanalysis », Educational Researcher, vol. 74, n 1, p. 29-58.

BAZERMAN C. (1981) : «What written knowledge does: three examples of academic discourse», Philosophy of the Social Sciences, vol. 11, p. 361-388.

BAZERMAN C. (1985) : «Physicists reading physics -schema-laden purposes and purpose-laden schéma», Written Communication, vol. 2, p. 3-23.

BAZERMAN C. (1988) : Shaping written knowledge: the genre and activity of the experimental article in science, Madison, University of Wisconsin Press.

Berkenkotter C. et Huckin T. (1995) : Genre knowledge in disciplinary communities: cognition/culture/power, Mahwah, NJ, Lawrence Erlbaum Associates.

BLoom B. S. (1956) : Taxonomy of educational objectives, Handbook I: the cognitive domain, New York, David McKay Co Inc.

Bосн F. (1999): La tâche d'écriture et de réécriture à l'université - la prise de notes, entre texte source et texte cible, Villeneuve-d'Ascq, Presses universitaires du Septentrion.

Boch F. et Grossmann F. (éds) (2001) : «De l'usage des citations dans le discours théorique : des constats aux propositions didactiques», Lidil, $\mathrm{n}^{\circ} 24$, p. $91-112$.

Boyer C. et Delbrayelle A. (2009) : «Épreuves en mathématiques et en français à l'école maternelle : quelles performances?», Les cahiers Théodile, $\mathrm{n}^{\circ}$ 10, p. 51-71. 
CARTer M. (1990) : «The idea of expertise: an exploration of cognitive and social dimensions of writing», CCC, vol. 41, p. 265-86.

Daunay B. (2002) : Éloge de la paraphrase, Presses universitaires de Vincennes, collection «Essais et savoirs ».

Delambre I. (2001) : «Formes diverses d'articulation entre discours d'autrui et discours propre; analyses de commentaires de textes théoriques », Lidil, n $^{\circ} 24$, p. 135-166.

Donahue C. (2002) : «Quelles stratégies pour mieux aider l'étudiantécrivain à gérer la polyphonie énonciative invitée par un travail avec d'autres textes?»,Enjeux, n 54, p. 67-80.

Donahue C. (2005) : «Student writing as negotiation: fundamental movements between the common and the specific in French essays », dans Fillia Kostouli (éd.), Writing in context(s):textual practices and learning processes in sociocultural settings, Amsterdam, Kluwer Academic Publishers.

Donahue C. (2007) : «Reformuler la reformulation : reprises-modification, négociation, et literate arts, vus dans des textes d'étudiants en première année universitaire», Recherches linguistiques, $\mathrm{n}^{\circ}$ 29, p. 180-192.

DONAHUE C. (2008) : Écrire à l'université : analyse comparée en France et aux États-Unis, Villeneuve-d'Ascq, Presses universitaires du Septentrion.

FABRe-Cols C. (2001) : «Trouver sa voix dans la rumeur des discours : accès au savoir et paroles empruntées au cours de la genèse du mémoire professionnel en IUFM», Lidil, n² 24, p. 49-70.

Fløтtum K. (2008) : «Standardisation versus diversification dans les écrits scientifiques: une perspective comparative de langues et de disciplines », Les discours universitaires : formes, pratiques, mutations (conférence, Bruxelles, Belgique).

FrançOIs F. (2009) : Essais sur quelques figures de l'orientation, Limoges, Lambert-Lucas.

Gannett C. (2008) : Proceed with caution: working with and working on inter-trans-cross national-institutional-global-cultural Writing Research, CCCC, LA, La Nouvelle-Orléans.

GeISLER C. (1994) : Academic literacy and the nature of expertise, Mahwah, NJ, Lawrence Erlbaum Associates.

Guibert R. (2001) : «Citer et se situer. L'apprentissage de l'écriture avec les discours d'autrui », Lidil, n² 24, p. 29-48.

Huortshoi K. (2001): The transition to college writing, NY, Bedford St. Martin's.

HowARD R. (1999) : Standing in the shadow of giants: plagiarists, authors, collaborators, Stamford, CT, Ablex. 
HyLAND K. (1996) : «Talking to the academy: forms of hedging in science research articles », Written Communication, vol. 13, p. 251-281.

LANGER J. A. (1992) : «Speaking of knowing: conceptions of understanding in academic disciplines », dans A. Herrington et C. Moran (éds), Writing, teaching, and learning in the disciplines, NY, Modern Language Association, p. 69-85.

LAVE J. et Wenger E. (1991) : Situated learning: legitimate peripheral participation, Cambridge, Cambridge University Press.

Monroe J. (2002) : Writing and revising the disciplines, Ithaca, NY, Cornell University.

PRIOR P. (1997) : «Literate activity and disciplinarity», Mind, culture, and activity, vol. 4, p. 275-295.

PRIOR P. (1998) : Writing/disciplinarity: a sociohistoric account of literate activity in the academy, Mahwah, NJ, Lawrence Erlbaum Associates.

Prior P. et Bazerman C. (2004) : What writing does and how it does it, Mahwah, NJ, Lawrence Erlbaum Associates.

Reuter Y. (2001) : «Je suis comme un autrui qui doute. Le discours des autres dans l'écrit de recherche en formation », Lidil, $\mathrm{n}^{\circ} 24$, p. 13-28.

Reuter Y. et Lahanier-Reuter D. (2008) : «Presentation of a few concepts for analyzing writing in relation to academic disciplines », L1 Educational studies in language and littérature, vol. 8, $\mathrm{n}^{\circ}$ 2, p. 47-57.

RINCK F. (2006) : «Gestion de la polyphonie et figure de l'auteur dans les parties théoriques de Rapports de stage » [en ligne], Lidil, $\mathrm{n}^{\circ} 34$. Disponible sur : <http://lidil.revues.org/index23.html> [consulté le 3 avril 2010].

Rinck F., Boch F. et Grossmann F. (2007) : «Quelques lieux de variation du positionnement énonciatif dans l'article de recherche», dans P. Lambert, A. Millet, M. Rispail et C. Trimaille (dir.), Variations au cour et aux marges de la sociolinguistique : mélanges offerts à Jacqueline Billiez, Paris, L'Harmattan, collection «Espaces discursifs », p. 285-296.

Russell D. et YANEZ A. (2003) : «Big picture people rarely become historians: genre systems and the contradictions of general education » [en ligne], dans C. Bazerman et D. Russell (éds), Writing selves/writing societies: research from activity perspectives, Fort Collins, Colorado, The WAC Clearinghouse and Mind, Culture, and Activity, p. 331-362. Disponible sur le site Internet du WAC Clearinghouse : <http://wac. colostate.edu/books/selves_societies/russell/russell.pdf $>$ [consulté le 14 avril 2010].

RussEll D. (1990) : «Writing across the curriculum in historical perspective», College English, vol. 52, n 1, p. 52-73. 
RuSSELl D. (1997) : «Rethinking genre in school and society», Written Communication, vol. 14, $\mathrm{n}^{\circ} 4$, p. 504-554.

Salomon G. et Perkins D. N. (1989) : «Rocky roads to transfer: rethinking mechanisms of a neglected phenomenon », Educational psychologist, vol. 24, $\mathrm{n}^{\circ} 2$, p. 113-142.

Sommers N. et SAltz L. (2004) : «The novice as expert: writing the freshman year», $C C C$, vol. 56, n 1 , p. 124-149.

SwAles J. (1990): Genre analysis: English in academic and research settings, Cambridge, Cambridge University Press.

Tuomi-Grohn T. et Engestrom Y. (2003) : «Conceptualizing transfer: from standard notions to developmental perspectives », dans T. Tuomi-Grohn et Y. Engestrom (éds), Between school and work: new perspectives on transfer and boundary-crossing, New York, Pergamon, p. 19-38.

Wenger E. (1999) : Communities of practice. learning, meaning and identity, Cambridge, Cambridge University Press.

\section{ANNEXE}

\section{Allen (Philosophie)}

Ses représentations à partir des entretiens :

- l'écrit est un mode d'enquête «philosophique»;

- le rôle de la lecture est essentiel;

- l'appropriation, l'intégration des lectures dans son système de savoirs est important;

- l'écrit dans la philosophie est perçu comme moyen d'évoluer, d' «aller plus loin».

Ses textes : recherches

Première année :

- il structure ses écrits dans des formes toutes faites telles des comparaisons traditionnelles;

- le discours d'autrui est fondamental;

- il y a peu de citations mais beaucoup de références.

\section{Quatrième année :}

- l'idée force est complexe;

- l'usage des sources est sophistiqué;

- la syntaxe est complexe;

- les notes de bas de page sont introduites.
Ses textes : analyses

Première année :

- les écrits travaillent autour d'un seul document de référence;

- ce document est mis en avant comme «source d'autorité».

\section{Quatrième année :}

- on trouve beaucoup de références et moins de citations, comme en première année dans les textes de recherches; - la syntaxe est complexe;

- les notes de bas de page sont introduites. 


\section{Joëlle (Biologie et Sciences Politiques «mineure»)}

Ses perceptions à partir des entretiens :

- la focalisation est sur le savoir tel que représenté et compris dans différentes disciplines;

- elle reconnait que les conventions dans les disciplines sont différentes (formats, formes de citation...);

- elle comprend l'écrit comme préparation à l'oral ou mode alternatif à l'oral.

Ses textes : recherches

Première année :

- elle écrit des «lab report» : genre très normé, écriture qui est formée par le genre;

- dans ses écrits on trouve une source référencée, plus le rapport de l'expérience scientifique.

\section{Quatrième année :}

- elle utilise une sélection plus étendue de sources, en synthèse;

- on trouve une fréquence élevée de marqueurs tels «pendant», «par exemple », «ainsi », «donc»;

- mais la tendance dominante reste celle d'une synthèse des autres plus que d'une présentation de nouvelles interprétations ;

- la voix passive prime, même dans l'idée force.
Ses textes : analyses

Première année :

- l'écrit reproduit des savoirs appris dans le cours et à partir des lectures;

- les perspectives des auteurs lus sont présentées comme «sans doute valables»;

- l'alternance entre «ils disent»«je dis» est formatrice.

\section{Quatrième année :}

- plutôt que «l'auteur dit» on trouve «l'auteur crée, donne son avis, maintient...»;

- le texte termine avec le point de vue de l'étudiant et non de l'auteur lu;

- on trouve davantage de connaissances représentées autour des thèmes traités.

\section{Robert (Psychologie et Sciences Politiques «double majeure»)}

Ses perceptions à partir des entretiens :

- il détient une perspective épistémologique sur l'écrit («cela sert à construire mes savoirs »);

- au départ, on trouve une reconnaissance des exigences universitaires : il faut «savoir de quoi on parle»;

- les écrits longs sont perçus comme plus exigeants;

- il évoque une façon de travailler qui permet de «mettre ses savoirs» sur papier;

- l'écrit est ce qui le pousse à «aller plus loin» dans sa pensée ;

- pour lui, il n'est plus question de faire simplement parce que le cours exige/le professeur exige...;

- également, il existe des normes «non-dites». 


\begin{tabular}{|c|c|}
\hline $\begin{array}{l}\text { Ses textes : recherches } \\
\text { De la première à la quatrième année, sa } \\
\text { façon de s'organiser, son point de vue } \\
\text { et autorité, et ses interactions avec les } \\
\text { sources changent de façon frappante. } \\
\text { Première année : } \\
\text { - il résume source après source de } \\
\text { façon linéaire; } \\
\text { - il choisit plutôt le verbe «être», } \\
\text { modalisé («semble être», par } \\
\text { exemple); } \\
\text { - plus de 50\% du texte est cité. } \\
\text { Quatrième année : } \\
\text { - il rédige des projets de recherches } \\
\text { interdisciplinaires; } \\
\text { - il construit des textes de «recherches» } \\
\text { dans le sens expérience-analyse qui } \\
\text { produit des savoirs; } \\
\text { - son écrit de fin d'études («senior } \\
\text { thesis») est une analyse de ses } \\
\text { propres écrits afin d'analyser les } \\
\text { influences des textes lus; } \\
\text { - ses textes se construisent avec les } \\
\text { caractéristiques scientifiques de } \\
\text { problématique, hypothèse, méthodo- } \\
\text { logie, tableaux, résultats...; } \\
\text { - le «senior thesis» est hybride : un } \\
\text { mélange de ces aspects scientifiques } \\
\text { avec des aspects interprétatifs plus } \\
\text { souvent associés aux humanités } \\
\text { (personnalisation de l'expérience, } \\
\text { par exemple). }\end{array}$ & $\begin{array}{l}\text { Ses textes : analyses } \\
\text { Première année : } \\
\text { - l'exemple d'une analyse } \\
\text { effectuée en cours d'histoire } \\
\text { d'un texte littéraire historique } \\
\text { souligne sa tendance à écrire } \\
\text { des phrases courtes, à utiliser } \\
\text { la voix passive, à introduire } \\
\text { des notes de bas de page. } \\
\text { Quatrième année : } \\
\text { - pour cet étudiant, on voit peu } \\
\text { d'évolution; } \\
\text { - les mêmes caractéristiques } \\
\text { qu'en première année, à part } \\
\text { des phrases d'introduction- } \\
\text { annonce, telles : « Je vais } \\
\text { comparer...» }\end{array}$ \\
\hline
\end{tabular}

